(2) Open Access Full Text Article

REVIEW

\title{
Improving patient outcomes in psoriasis: strategies to ensure treatment adherence
}

\author{
This article was published in the following Dove Press journal: \\ Psoriasis: Targets and Therapy \\ 17 July 2015 \\ Number of times this article has been viewed
}

\section{Oriol Yélamos \\ Sandra Ros \\ Lluís Puig}

Department of Dermatology, Hospital de la Santa Creu i Sant Pau -

Universitat Autònoma de Barcelona, Barcelona, Catalonia, Spain
Correspondence: Lluís Puig Department of Dermatology, Hospital de la Santa Creu i Sant Pau - Universitat Autònoma de Barcelona, C/Sant Antoni Maria Claret 167, 08025 Barcelona, Spain Tel +34 935537007

Fax +34935537008

Email lpuig@santpau.cat
Abstract: Psoriasis is a frequent inflammatory disease with a chronic and relapsing course. Therefore, patients with psoriasis are likely to undergo different treatments for long periods of time. Traditionally, therapies used in psoriasis have been associated with poor levels of adherence due to the complexity of the regimens and the poor results obtained with the topical therapies. These poor outcomes are associated with high levels of frustration and anxiety, which decrease adherence and worsen the disease. With the recent introduction of highly efficacious biologic therapies, patients can achieve very good and prolonged responses. However, most patients with psoriasis have mild disease and may be treated with skin-directed therapies. Therefore, it is important to develop strategies to improve adherence in order to achieve better outcomes, and to improve the overall quality of life. Hence, acknowledging the causes of nonadherence is crucial for implementing these strategies. In this summary, we review the causes of nonadherence, and we provide behavioral strategies in order to improve adherence and, ultimately, the outcome of patients with psoriasis.

Keywords: psoriasis, adherence, outcome, drug therapy, psychotherapy

\section{Introduction}

Psoriasis is a frequent inflammatory disease affecting approximately $2 \%$ of the population in western countries. ${ }^{1}$ Psoriasis can affect either young or elderly people, and it has a chronic and relapsing course. Therefore, patients with psoriasis are likely to undergo different treatments for long periods of time. Traditionally, therapies used in psoriasis have been associated with poor levels of treatment adherence due to the complexity of the regimens and the poor results obtained with topical therapies. ${ }^{2}$ These poor outcomes are associated with high levels of frustration, anxiety, and depression, which worsen the disease due to both a direct effect of stress in the immune system ${ }^{3}$ and a decrease in treatment adherence. With the recent introduction of highly efficacious biologic therapies, patients can achieve very good and prolonged responses. However, most patients with psoriasis have mild disease and may be treated more likely with skin-directed therapies.

Therefore, it is crucial to develop strategies to improve adherence in order to achieve better outcomes, prevent flares, control the comorbidities associated with psoriasis, and improve the overall quality of life (QoL) of patients with psoriasis. Hence, acknowledging the causes of nonadherence is fundamental to be able to implement these strategies. In the present article, we will review the causes of nonadherence and provide strategies to improve treatment adherence in patients with psoriasis. 


\section{Methods}

We have performed a literature search in Medline using the keywords "(psoriasis OR dermatology) AND (adherence OR compliance)". This search prompted 1,627 articles that were selected according to their relevance by reviewing the available abstracts. Articles were selected if they provided clear definitions on terms such as adherence and compliance, or if they reviewed factors that influence or modify adherence. We also searched for specific keywords such as adherence, compliance, persistence, psychological impact, and psychological evaluation in psoriasis. In total, 75 articles were read and reviewed and, finally, 49 were included in the current manuscript. We have classified the causes of nonadherence in three categories, depending on if they were linked with factors related to the therapies, factors related to the patient, and factors related to the patient-physician relationship. For each of these categories, measures to increase adherence were also suggested.

\section{Adherence in psoriasis}

Adherence is a complex human behavior modulated by subjective components. As the name suggests, adherence is associated with being voluntarily attached to something in a constant manner. ${ }^{4}$ Generally, adherence is poorer in chronic diseases, with treatments that are difficult to apply, with preventive measures, or preventive strategies. Thus, it is easy to understand why adherence in dermatology is poor, as it is due to the relapsing nature of the most common skin diseases and the use of multiple topical treatments which, in most cases, are not applied correctly. In fact, Storm et al ${ }^{5}$ noted that $95 \%$ of patients with skin problems that were seen for the first time in a dermatology department underdosed their topical therapies. Patients with psoriasis are not an exception and show poor adherence to treatments. ${ }^{2}$

Nonadherence is a major economical concern, as it associated with more costs, both direct and indirect. Bad adherence in psoriasis is associated with worse disease control and it may require the use of more potent and expensive drugs; it may also have a direct impact on societal productivity. Patients with a bad control of their psoriasis will require more appointments with their health care providers, and therefore will be more prone to losing job days and being less productive. Thus, improving adherence is a must, as strategies that improve adherence are related to a better control of the disease. ${ }^{6}$

Nonadherence can be divided into intentional and unintentional, depending on which motivations lead the patient to not follow a certain therapy. ${ }^{7}$ Unintentional nonadherence is mainly related to problems with applying treatments, errors in understanding the prescription, and forgetting to apply or take medications. Conversely, intentional nonadherence occurs when patients deliberately decide not to continue on a certain therapy. This can be due to preexisting psychopathology, although it is most frequently due to communication problems between patients and their physicians or due to poor results with their current therapies. ${ }^{8}$ Nonadherence can also be divided into primary and secondary. Primary nonadherence refers to not redeeming prescriptions and not starting a treatment, whereas secondary nonadherence refers to not using the medications correctly. ${ }^{9}$ An additional concept linked to secondary nonadherence is persistence, which refers to the continuous use of a treatment and not stopping it earlier than prescribed..$^{10}$ In psoriasis, both primary and secondary nonadherence are poor, with less than $50 \%$ of patients redeeming their prescriptions ${ }^{11}$ and only one- to two-thirds of patients using the medication correctly. ${ }^{11-15}$

Hence, adherence is influenced by multiple behavioral factors. In addition, psoriasis is a condition with a very important psychosomatic component, which can worsen the disease when high levels of stress are present, due to poor adherence but also due to a direct effect of stress on the outcome of the disease. ${ }^{16}$ Therefore, in patients with suspected poor adherence or poor outcomes, it can be useful to evaluate the psychological impact of psoriasis.

\section{Psoriasis, psychological impact, and influence on treatment adherence}

Psoriasis is associated with a strong psychological impact due to its visibility and a chronic and relapsing course. ${ }^{17-19}$ The disfigurement in psoriasis decreases self-esteem, ${ }^{16}$ and induces the onset of stigmatization and the anticipation to rejection..$^{20}$ This is associated with high stress levels, anxiety, depressive symptoms, and changes in behavior, such as toxic habits or poor adherence to treatments. ${ }^{12,17,21}$ Stress increases proinflammatory cytokines, which interact with the lymphocytes, and produces a suppressive effect in the immune system. ${ }^{3}$ At the same time, these cytokines induce depressive symptoms and anxiety, which can trigger psoriasis flares and also reduce adherence. Thus, psoriasis is a good example of a psychosomatic disease, ${ }^{16}$ as psoriasis increases the levels of stress and anxiety and, at the same time, stress worsens the clinical course of the disease. Therefore, an early evaluation and treatment of the psychological impact in patients with psoriasis is crucial to implement strategies to improve adherence to treatment and ultimately improve the clinical outcome. 
Several tests are currently available to evaluate the psychological impact of psoriasis. As previously stated, QoL can be severely decreased in patients with psoriasis. In order to evaluate QoL, the Dermatology Life Quality Index (DLQI) ${ }^{22}$ is the most commonly used QoL evaluation tool in psoriasis. However, the results can vary between countries due to cultural differences. Therefore, the use of an additional test may be useful in patients with important psychological burden. A good complementary test is Skindex 16 that, on one page, includes 16 items that evaluate how much patients are bothered by their skin conditions. ${ }^{23}$ The evaluation of QoL is fundamental, as very impaired QoL may be associated with poor adherence and poor outcomes and may be the indication for biologic drugs. ${ }^{24-26}$

In addition, in select patients, it may be useful to screen for potential psychiatric disorders such as depression, anxiety, and alcohol abuse, which are associated with poor adherence. The prevalence of depression in patients with psoriasis is approximately $30 \%$, with up to $10 \%$ of patients reporting suicidal ideation. ${ }^{27}$ Therefore, it is crucial to ask about depressive symptoms in patients with psoriasis. It is especially important to investigate the presence of anhedonia (not being capable of enjoying those things that are commonly pleasurable), as this is probably the most useful clue to screen for depression. In cases with suspected depression, good screening tests include the Hospital Anxiety and Depression Scale (HADS) ${ }^{28}$ and the Goldberg Depression and Anxiety Scale. ${ }^{29}$

Eventually, patients with psoriasis may have increased their consumption of alcohol, especially if their psoriasis is moderate or severe, and this is associated with poor adherence. ${ }^{30}$ Therefore, to screen for alcohol misuse, we recommend using the Alcohol Use Disorders Identification Test (AUDIT), which is a simple ten-item test with high sensitivity and specificity to detect harmful alcohol use. ${ }^{31}$ Regardless of the health benefits associated with alcohol cessation in patients with heavy alcohol use, in the specific setting of psoriasis, alcohol cessation is especially relevant, as alcoholism is associated with worse outcomes, worse adherence to treatment, more severe disease, ${ }^{30}$ increased risk of fatty liver (which may limit the use of certain drugs such as methotrexate), ${ }^{32}$ and even increased risk of mortality. ${ }^{33}$

Hence, in patients with suspected poor adherence, it may be advisable to evaluate their QoL and to screen for depression, anxiety, and heavy alcohol use. If this evaluation disclose psychosocial comorbidities, patients should be referred to a psychologist or psychiatrist in order to improve adherence, and also to screen for psychopathological comorbidities. ${ }^{34}$ However, certain situations may require professional psychological evaluation regardless of the test results, such as adjustment disorders, suicidal ideation, onset of the disease related to stress in adolescents or young adults, poor clinical response due to bad adherence, and extreme concern in relation to the severity of the psoriasis. ${ }^{17,34}$

\section{Causes of nonadherence and measures to increase adherence}

Despite the impact of psychosomatic comorbidities, other causes can also influence adherence in patients with psoriasis. From a practical standpoint, nonadherence can be influenced by factors related to patient characteristics and beliefs, patient-physician relationship, and treatment. ${ }^{12}$ All of these factors should be acknowledged and identified by health care providers to implement strategies to increase adherence, maintain long-term persistence, and ultimately improve the outcome of patients with psoriasis. Although some of these factors cannot be changed, identifying individuals at a higher risk of nonadherence can be beneficial when deciding the best therapeutic approach for each patient. In subsequent sections, and in Table 1, we summarize the causes of nonadherence, and we discuss possible strategies to improve adherence in patients with psoriasis.

Table I Strategies to improve adherence in patients with psoriasis

Strategies related to the patient

- Health education

- To the patient

- Public campaigns

- Alcohol/tobacco cessation

- Psychotherapy

- Relaxation techniques

- Disease coping techniques

- Support groups

- Psychiatry referral if psychopathology

Strategies related to the patient-physician relationship

- Pleasant and friendly attitude

- Visual contact

- Empathy

- Trusting relationship with the patient

- Active listening

- Agreed therapeutic regimens

- Avoidance of medical jargon

Strategies related to the therapy

- Feasible to apply in patients with extensive involvement

- High efficacy

- Low cost

- Nongreasy, clean, nonsmelling, nonstaining (gels, foams, lotions)

- Infrequent application/intake (weekly, monthly)

- Minimal adverse effects

- Written instructions 


\section{Factors related to the patient}

Certain demographic factors have been associated with worse adherence and include male sex, unemployment, singleness, and heavy alcohol and tobacco consumption. ${ }^{35}$ Adherence is also poorer in patients with severe psoriasis compared to patients with mild psoriasis. ${ }^{35}$ These patients have a worse psychological impact and may not rely on therapies, leading to a decrease in adherence. ${ }^{12,36}$ The more severe impact of psoriasis is that it also determines lower salaries, as patients with visible psoriasis will not be hired for certain positions, or they will not even apply for certain jobs. In addition, the low income associated with this situation will have a negative impact on adherence, as some patients will not be able to afford expensive medications. ${ }^{17}$ Hence, public campaigns to raise awareness of psoriasis can be performed in order to eliminate the stigmatization associated with the disease and to provide more equal opportunities for all the individuals in society.

In addition, patients that have a limited income, live alone, or engage in heavy alcohol consumption are more likely to have anxious and depressive symptoms, which will worsen their psoriasis and make it difficult to gain adequate control of the disease. Thus, in patients with psoriasis, it is fundamental to identify the presence of associated psychopathology or substance abuse using the aforementioned tools in order to provide adequate referral and treatment. Also, in patients with high stress levels and poor social support, psychotherapy has proven to be effective in order to improve adherence. ${ }^{37-39}$ Psychological strategies that may be useful include relaxation techniques, strategies to cope with the disease, and support groups. In select cases with severe psychopathology, the use of psychoactive drugs may also be beneficial. ${ }^{40}$

Coping with the disease is fundamental to improving adherence. Initially, when psoriasis is diagnosed, most patients are not fully aware of the chronic and relapsing course of the disease. Thus, it is fundamental to give adequate information about the disease, focusing on the chronic nature of psoriasis and that the treatment goal is controlling the symptoms rather than curing the disease. Also, patients should be informed that therapies will likely be used for prolonged periods of time. This will give realistic expectations to the patients about what to expect with their therapies and the drug-related side effects. Ultimately, this approach will prevent patients from developing maladaptive behaviors such as denial of the disease or fear of side effects, which may result in bad adherence. ${ }^{41}$

Forgetting to take medications is also a main issue related to poor adherence, and several strategies can be used as reminders, such as text messages, ${ }^{8}$ emails, or phone calls. In that sense, nurses are being increasingly more involved in becoming a key part of the treatment of patients with psoriasis, ${ }^{42}$ not only by helping them with treatment reminders but also by helping patients when they have doubts about how to apply the medication, and by giving important information about the disease.

\section{Factors related to the patient-physician relationship}

In a recent study, patients with psoriasis stated that they considered their disease dirty, and that this was not taken into account by their physicians. ${ }^{8}$ In addition, patients considered that their physicians did not pay enough attention to prescribing adequate treatments for their disease. Although these perceptions are not always true, an optimal relationship between the patient and his or her health care providers is fundamental to enhance adherence. ${ }^{43,44} \mathrm{~A}$ bad relationship between the patient and his or her physicians may lead to a loss of trust toward medications and health care providers, and it has a negative impact on adherence. An important percentage of patients may have unrealistic expectations related to therapy, and therefore physicians should provide realistic therapeutic goals to their patients. ${ }^{8}$

It is also important to identify certain patterns associated with adherence in patients with psoriasis. Adherence improves in the days before and after the appointment with the physician. ${ }^{45}$ Therefore, performing an early follow-up visit with the patient is an excellent opportunity to improve the patient-physician relationship and to increase adherence. ${ }^{12}$ However, a recent study showed that not a lot of physicians use this strategy, which is obviously a missed opportunity to improve adherence in dermatologic conditions. ${ }^{46}$

Another issue that decreases adherence is not considering patients' beliefs, expectations, or preferences. ${ }^{8}$ Therefore, agreeing to and reassuring the therapy and the regimen with the patient according to his or her preferences is fundamental to increasing treatment adherence ${ }^{47}$ Therefore, paternalistic or defensive attitudes should be avoided, as they will worsen the relationship between the patient and the physician. In addition, certain strategies can be implemented to improve this relationship, such as avoiding the use of medical jargon, showing a pleasant and empathetic attitude toward the patient, and providing a cooperative environment in order to discuss the best treatment options for each patient.

\section{Factors related to the treatment}

Nonadherence related to treatment is associated with a variety of factors. Factors associated with negative opinions 
regarding treatments include greasy vehicles, excessive time needed to apply the treatment, fear of side effects, and slowacting treatments..$^{8,12,14,17,21}$ For example, topical treatments are less preferred than systemic drugs or phototherapy, but they are considered safer. ${ }^{48}$ Overall, phototherapy and injectable drugs are treatments with higher patient satisfaction and adherence, not only because these treatments are more effective but also because patients are more involved in their treatments. ${ }^{49}$ In fact, making patients become more involved in their therapies has been associated with increased levels of adherence. ${ }^{44,50}$

However, differences exist within the same therapeutic modality. For example, within topical corticosteroids, the results obtained can vary depending on different factors, such as potency of the corticosteroid, the location where the treatment is applied, or the vehicle used. Regarding this last factor, traditional dermatologic knowledge has highlighted that thick vehicles such as ointments have better efficacy than lighter formulations. However, it has been shown that efficacy rates are similar between ointments and other formulations, such as creams, foams, shampoos, or lotions. ${ }^{51}$ In addition, the use of more cosmetic vehicles such as gels is preferred by patients and is associated with better adherence. ${ }^{52}$ Ideally, a topical treatment should be nongreasy, efficacious, safe, and feasible to apply to different anatomic areas. This is especially important in areas such as the scalp, where the application of foams, shampoos, or gels is preferred instead of the use of greasier vehicles. ${ }^{52}$ In fact, certain locations, such as the scalp or the nails, can be very troublesome because the lesions are highly visible and the application of topical treatments is difficult requiring, in some cases, the use of systemic therapies. ${ }^{52-55}$ Nevertheless, the decision as to which vehicle should be used should also rely on the patients' preferences, as this will ultimately affect adherence. ${ }^{51}$

Another important factor to consider is the treatment regimen. In a survey where patients with psoriasis were asked about the reasons for not adhering to treatments, the most frequent answer was that treatment had to be applied too often. ${ }^{12}$ Therefore, simplifying the treatment ${ }^{21}$ and choosing stronger therapies with less frequent regimens is a good strategy to improve adherence. However, a special comment has to be made regarding treatments that are not applied or taken daily, such as systemic methotrexate or biologics. In these cases, ensuring that the patient understands the prescription, and that he or she is also given clear written or even graphical instructions on treatment regimens, can be helpful to improve adherence and to prevent the development of side effects which, in certain circumstances, can be life threatening. ${ }^{32,56}$

\section{Discussion}

When poor adherence is suspected, it is important to ask for the patients' preferences regarding different treatment options, as well as to explore the social background of the patient (social support, economic situation, substance abuse), and also to screen for the presence of anxiety or depressive symptoms. If nonadherence is noted, it is important to identify if nonadherence is intentional or unintentional, and not to blame the patient because this will further decrease adherence and final outcomes. On the other hand, discussing the reasons for not adhering to a therapy is a better approach if we want our patients to successfully use the prescribed therapies.

Even though psychological factors and personality traits are always important, the reasons for inadequate adherence in psoriasis differ according to the type of treatment. With respect to topical treatment, the main reasons for poor adherence are perceived lack of efficacy, slow onset of effect, and inconvenience of application, due to its frequency and vehicle characteristics. ${ }^{57}$ In the case of conventional systemic drugs, the occasional skipping of doses is unlikely to be clinically relevant, but treatment discontinuation is generally associated with tolerability/safety issues $(43 \%)$ or a lack/loss of effectiveness $(30 \%){ }^{58}$ The main reason for nonfulfillment of biologic prescriptions (primary nonadherence) is concern about potential adverse effects and safety issues, and discontinuation (secondary nonadherence) is due to tolerability/safety problems (25\%), a lack or loss of effectiveness $(22 \%)$, injection reactions or inconvenience $(10 \%)$, and cost/ insurance reimbursement issues $(11 \%) .{ }^{58}$ Treatment cessation because of an adequate response cannot be considered a lack of adherence, since the physician and/or the patient consider the treatment is no longer needed; this constitutes the basis for intermittent therapy. The same applies to dosage individualization through the lengthening of administration intervals, which can be decided by the prescriber or the patient on his or her own accord, but it cannot be considered a lack of adherence since the therapeutic goal is maintained.

\section{Conclusion}

To sum up, psoriasis is a disease with an important psychosomatic background, in which stress plays an important role, not only with the onset of acute flares but also in the presence of associated comorbidities. The prevalence of psychological comorbidities is high and may require referral to a psychologist or psychiatrist if psychopathology is noted. Moreover, in order to improve adherence, a good relationship with patients is crucial, showing an empathetic approach toward our patients. A joint decision regarding which therapies 
will be better in each case is essential to guaranteeing a successful outcome in our patients. Choosing therapies that are efficacious, safe, and easy to apply will enhance adherence. However, in some cases, a multidisciplinary, holistic approach involving psychologists, psychiatrists, rheumatologists, nurses, and other health care providers may be necessary.

\section{Disclosure}

The authors report no conflicts of interest in this work.

\section{References}

1. Boehncke WH, Katsambas A, Ortonne JP, Puig L. EADV preceptorship: advances in dermatology. JEur Acad Dermatol Venereol. 2010;24 Suppl 5: $2-24$.

2. Ali SM, Brodell RT, Balkrishnan R, Feldman SR. Poor adherence to treatments: a fundamental principle of dermatology. Arch Dermatol. 2007;143(7):912-915.

3. Chapman BP, Moynihan J. The brain-skin connection: role of psychosocial factors and neuropeptides in psoriasis. Expert Rev Clin Immunol. 2009;5(6):623-627.

4. Aronson JK. Compliance, concordance, adherence. Br J Clin Pharmacol. 2007;63(4):383-384.

5. Storm A, Benfeldt E, Andersen SE, Serup J. A prospective study of patient adherence to topical treatments: $95 \%$ of patients underdose. $\mathrm{JAm}$ Acad Dermatol. 2008;59(6):975-980.

6. Carroll CL, Feldman SR, Camacho FT, Balkrishnan R. Better medication adherence results in greater improvement in severity of psoriasis. Br J Dermatol. 2004;151(4):895-897.

7. Atkins L, Fallowfield L. Intentional and non-intentional nonadherence to medication amongst breast cancer patients. Eur J Cancer. 2006;42(14):2271-2276.

8. Bewley A, Burrage DM, Ersser SJ, Hansen M, Ward C. Identifying individual psychosocial and adherence support needs in patients with psoriasis: a multinational two-stage qualitative and quantitative study. J Eur Acad Dermatol Venereol. 2014;28(6):763-770.

9. Anderson KL, Dothard EH, Huang KE, Feldman SR. Frequency of primary nonadherence to acne treatment. JAMA Dermatol. 2015; 151(6):623-626

10. Kardas P, Lewek P, Matyjaszczyk M. Determinants of patient adherence: a review of systematic reviews. Front Pharmacol. 2013;4:91.

11. Storm A, Andersen SE, Benfeldt E, Serup J. One in 3 prescriptions are never redeemed: primary nonadherence in an outpatient clinic. $J A m$ Acad Dermatol. 2008;59(1):27-33.

12. Bewley A, Page B. Maximizing patient adherence for optimal outcomes in psoriasis. J Eur Acad Dermatol Venereol. 2011;25 Suppl 4:9-14.

13. Richards HL, Fortune DG, O'Sullivan TM, Main CJ, Griffiths CE. Patients with psoriasis and their compliance with medication. $J \mathrm{Am}$ Acad Dermatol. 1999;41(4):581-583.

14. Fouéré S, Adjadj L, Pawin H. How patients experience psoriasis: results from a European survey. J Eur Acad Dermatol Venereol. 2005; 19 Suppl 3:2-6.

15. Augustin M, HollandB, DartschD, LangenbruchA, Radtke MA. Adherence in the treatment of psoriasis: a systematic review. Dermatology. 2011; 222(4):363-374.

16. Rieder E, Tausk F. Psoriasis, a model of dermatologic psychosomatic disease: psychiatric implications and treatments. Int J Dermatol. 2012; 51(1):12-26.

17. Ros S, Puig L, Carrascosa JM. Cumulative life course impairment: the imprint of psoriasis on the patient's life. Actas Dermosifiliogr. 2014; 105(2):128-134.
18. Bhatti ZU, Finlay AY, Bolton CE, et al. Chronic disease influences over 40 major life-changing decisions (MLCDs): a qualitative study in dermatology and general medicine. J Eur Acad Dermatol Venereol. 2014;28(10):1344-1355.

19. Bhatti ZU, Salek MS, Finlay AY. Major life changing decisions and cumulative life course impairment. J Eur Acad Dermatol Venereol. 2011;25(2):245-246; author reply 246.

20. Hrehorów E, Salomon J, Matusiak L, Reich A, Szepietowski JC. Patients with psoriasis feel stigmatized. Acta Derm Venereol. 2012; 92(1):67-72.

21. Richards HL, Fortune DG, Griffiths CE. Adherence to treatment in patients with psoriasis. J Eur Acad Dermatol Venereol. 2006; 20(4):370-379.

22. Finlay AY, Khan GK. Dermatology Life Quality Index (DLQI) a simple practical measure for routine clinical use. Clin Exp Dermatol. 1994;19(3):210-216.

23. Chren MM, Lasek RJ, Sahay AP, Sands LP. Measurement properties of Skindex-16: a brief quality-of-life measure for patients with skin diseases. J Cutan Med Surg. 2001;5(2):105-110.

24. Pathirana D, Ormerod AD, Saiag P, et al. European S3-guidelines on the systemic treatment of psoriasis vulgaris. $J$ Eur Acad Dermatol Venereol. 2009;23 Suppl 2:1-70.

25. Menter A, Korman NJ, Elmets CA, et al. Guidelines of care for the management of psoriasis and psoriatic arthritis: section 4 . Guidelines of care for the management and treatment of psoriasis with traditional systemic agents. $J$ Am Acad Dermatol. 2009; 61(3):451-485.

26. Samarasekera E, Sawyer L, Parnham J, Smith CH; Guideline Development Group. Assessment and management of psoriasis: summary of NICE Guidelines. BMJ. 2012;345:e6712.

27. Schmitt JM, Ford DE. Role of depression in quality of life for patients with psoriasis. Dermatology. 2007;215(1):17-27.

28. Zigmond AS, Snaith RP. The hospital anxiety and depression scale. Acta Psychiatr Scand. 1983;67(6):361-370.

29. Goldberg D, Bridges K, Duncan-Jones P, Grayson D. Detecting anxiety and depression in general medical settings. BMJ. 1988; 297(6653):897-899.

30. McAleer MA, Mason DL, Cunningham S, et al. Alcohol misuse in patients with psoriasis: identification and relationship to disease severity and psychological distress. Br J Dermatol. 2011;164(6): 1256-1261.

31. Babor TF, Higgins-Biddle JC, Saunders JB, Monteiro MG. Audit: The Alcohol Use Disorders Identification Test. Guidelines for Use in Primary Care. 2nd ed. Geneva, Switzerland: World Health Organization; 2001. Available from: http://whqlibdoc.who.int/hq/2001/ WHO_MSD_MSB_01.6a.pdf. Accessed April 2, 2015.

32. Yélamos O, Puig L. Systemic methotrexate for the treatment of psoriasis. Expert Rev Clin Immunol. 2015;11(5):553-563.

33. Poikolainen K, Karvonen J, Pukkala E. Excess mortality related to alcohol and smoking among hospital-treated patients with psoriasis. Arch Dermatol. 1999;135(12):1490-1493.

34. Janowski K, Pietrzak A. Indications for psychological intervention in patients with psoriasis. Dermatol Ther. 2008;21(5):409-411.

35. Zaghloul SS, Goodfield MJ. Objective assessment of compliance with psoriasis treatment. Arch Dermatol. 2004;140(4):408-414.

36. Kimball AB, Jacobson C, Weiss S, Vreeland MG, Wu Y. The psychosocial burden of psoriasis. Am J Clin Dermatol. 2005;6(6):383-392.

37. Fortune DG, Richards HL, Kirby B, Bowcock S, Main CJ, Griffiths CE. A cognitive-behavioural symptom management programme as an adjunct in psoriasis therapy. Br J Dermatol. 2002; 146(3):458-465.

38. Fordham B, Griffiths CE, Bundy C. Can stress reduction interventions improve psoriasis? A review. Psychol Health Med. 2013;18(5): 501-514.

39. Price ML, Mottahedin I, Mayo PR. Can psychotherapy help patients with psoriasis? Clin Exp Dermatol. 1991;16(2):114-117. 
40. D'Erme AM, Zanieri F, Campolmi E, et al. Therapeutic implications of adding the psychotropic drug escitalopram in the treatment of patients suffering from moderate-severe psoriasis and psychiatric comorbidity: a retrospective study. J Eur Acad Dermatol Venereol. 2014;28(2):246-249.

41. Kossakowska MM, Cieścińska C, Jaszewska J, Placek WJ. Control of negative emotions and its implication for illness perception among psoriasis and vitiligo patients. J Eur Acad Dermatol Venereol. 2010; 24(4):429-433.

42. Ersser SJ, Cowdell FC, Nicholls PG, Latter SM, Healy E. A pilot randomized controlled trial to examine the feasibility and efficacy of an educational nursing intervention to improve self-management practices in patients with mild-moderate psoriasis. J Eur Acad Dermatol Venereol. 2012;26(6):738-745.

43. Feldman SR. Approaching psoriasis differently: patient-physician relationships, patient education and choosing the right topical vehicle. J Drugs Dermatol. 2010;9(8):908-911.

44. Reich K, Mrowietz U, Karakasili E, Zschocke I. Development of an adherence-enhancing intervention in topical treatment termed the topical treatment optimization program (TTOP). Arch Dermatol Res. 2014;306(7):667-676.

45. Feldman SR, Camacho FT, Krejci-Manwaring J, Carroll CL, Balkrishnan R. Adherence to topical therapy increases around the time of office visits. J Am Acad Dermatol. 2007;57(1):81-83.

46. Davis SA, Lin HC, Yu CH, Balkrishnan R, Feldman SR. Underuse of early follow-up visits: a missed opportunity to improve patients' adherence. J Drugs Dermatol. 2014;13(7):833-836.

47. Ersser SJ, Surridge H, Wiles A. What criteria do patients use when judging the effectiveness of psoriasis management? J Eval Clin Pract. 2002;8(4):367-376.

48. van Cranenburgh OD, de Korte J, Sprangers MA, de Rie MA, Smets EM. Satisfaction with treatment among patients with psoriasis a web-based survey study. Br J Dermatol. 2013;169(2):398-405.

49. Baker CS, Foley PA, Braue A. Psoriasis uncovered - measuring burden of disease impact in a survey of Australians with psoriasis. Australias J Dermatol. 2013;54 Suppl 1:1-6.
50. Martin LR, Williams SL, Haskard KB, Dimatteo MR. The challenge of patient adherence. Ther Clin Risk Manag. 2005;1(3):189-199.

51. Warino L, Balkrishnan R, Feldman SR. Clobetasol propionate for psoriasis: are ointments really more potent? J Drugs Dermatol. 2006; 5(6):527-532.

52. Puig L, Ribera M, Hernanz JM, et al. [Treatment of scalp psoriasis: review of the evidence and Delphi consensus of the Psoriasis Group of the Spanish Academy of Dermatology and Venereology]. Actas Dermosifiliogr. 2010;101(10):827-846. Spanish.

53. Papp K, Berth-Jones J, Kragballe K, Wozel G, de la Brassinne M. Scalp psoriasis: a review of current topical treatment options. J Eur Acad Dermatol Venereol. 2007;21(9):1151-1160.

54. Ortonne J, Chimenti S, Luger T, Puig L, Reid F, Trüeb RM. Scalp psoriasis: European consensus on grading and treatment algorithm. J Eur Acad Dermatol Venereol. 2009;23(12):1435-1444.

55. Langley RG, Saurat JH, Reich K; Nail Psoriasis Delphi Expert Panel. Recommendations for the treatment of nail psoriasis in patients with moderate to severe psoriasis: a dermatology expert group consensus. J Eur Acad Dermatol Venereol. 2012;26(3):373-381.

56. Yélamos O, Català A, Vilarrasa E, Roé E, Puig L. Acute severe methotrexate toxicity in patients with psoriasis: a case series and discussion. Dermatology. 2014;229(4):306-309.

57. Puig L, Carrascosa JM, Belinchón I, et al; Panel de Expertos del Consenso Delphi sobre Tratamiento tópico de la psoriasis; Grupo de Psoriasis de la Academia Española de Dermatología y Venereología. Adherence and patient satisfaction with topical treatment in psoriasis, and the use, and organoleptic properties of such treatments: a Delphi study with an expert panel and members of the Psoriasis Group of the Spanish Academy of Dermatology and Venereology. Actas Dermosifiliogr. 2013;104(6):488-496.

58. Lebwohl MG, Bachelez H, Barker J, et al. Patient perspectives in the management of psoriasis: results from the population-based Multinational Assessment of Psoriasis and Psoriatic Arthritis Survey. J Am Acad Dermatol. 2014;70(5):871-881. e1-e30.
Psoriasis: Targets and Therapy

\section{Publish your work in this journal}

Psoriasis: Targets and Therapy is international, peer-reviewed, open access journal focusing on psoriasis, nail psoriasis, psoriatic arthritis and related conditions, identification of therapeutic targets and the optimal use of integrated treatment interventions to achieve improved outcomes and quality of life. The manuscript management system

\section{Dovepress}

is completely online and includes a very quick and fair peer-review system. Visit http://www.dovepress.com/testimonials.php to read real quotes from published authors. 